\title{
3D-Modeling and Motion Simulation of Composite Wheel Gears Transmission Based on UG
}

\author{
Mingxia Zhao ${ }^{1, a}$ \\ ${ }^{1}$ State Grid Jibei Electric Power Company Limited Skills Training Center, Baoding Electric Power VOC.\& TECH. College, 071000, \\ Baoding, China
}

\begin{abstract}
Taking the compound gear trains as an example, the principle of the transmission mechanism was analyzed, and the rotational speed of the key gears in the compound gear trains was calculated by using the calculation formula of transmission ratio to obtain the simulation parameters of UG movement. The gear tool box in UG was applied to complete the modeling and meshing assembly of the bevel gear and spur gear, the rotation pair and gear pair was to motion simulation, the gear transmission state could have visually observed by motion simulation, and then the chart was analyzed to verify the design rationality of the gear train.
\end{abstract}

\section{Introduction}

The UG software has been added NXGC toolbox since version 6.0 , which gear modeling toolbox can help users to accurately and quickly create involute spur, bevel or bevel gear, etc., but also for the gears' meshing and moving operations; so that it improves the design efficiency of gear train, and reduces the workload of designers. The motion simulation module is a CAE application software with powerful static and kinematic, kinematic analysis and calculation functions and dynamic simulation functions, which is used to establish the movement mechanism model to simulate the movement rule, and can perform interference analysis for the movement mechanism, track the movement trajectory of the parts or analysis the speed, acceleration, etc., of the institutional parts; furthermore, build independent motion analysis schemes without affecting the main assembly mode[1-2].

\section{Principle Analysis Of Compound Gear Train}

The gear train was a gear transmission system composed of a series of gears. The running of gear train could be divided into three categories according to whether the position of each gear axis relative to the rack was fixed or not: the fixed axis gear train, the epicyclical gear train and the compound gear train. Compound gear train in the actual use of machinery was the most common, which included both fixed axis gear train and epicyclical gear train; or was comprised by the several parts of epicyclical gear train[3].

A compound gear train mechanism was shown as figure 1 , which was the input speed as $\mathrm{W} 1=19 \mathrm{r} / \mathrm{min}$, and the number of gears as: $\mathrm{Z} 1=90, \mathrm{Z} 2=60, \mathrm{Z} 2^{\prime}=\mathrm{Z} 3=$
$30, Z^{\prime}=24, Z 4=18, Z 5=15, Z 5^{\prime}=30, Z 6=105, Z 8=$ $35, Z 7=32$. The compound gear train consisted of a fixed axis gear train and a epicyclical gear train, where a fixed axis gear train (a) was comprised of the bevel gear $1-2-2$ '-3, a epicyclical gear train (b) was formed by the gear 3'-4-5-5'-6, and a fixed axis gear train (c) was constituted by the gear 7-8.



Figure 1. An organization diagram of compound gear train

\section{Transmission calculation of mechanism for compound gear train}

For the fixed axis gear train, transmission ratio of each gear pair in the gear train was calculated as equation (1).

$$
i_{j, j+1}=\frac{W_{j}}{W_{j+1}}= \pm \frac{z_{j}}{z_{j+1}}
$$

As for the epicyclical gear train, planet gear had both rotation and revolution due to the rotation of the planet carrier, so that its transmission ratio could not be directly calculated by using the transmission ratio of the fixed axis gear train. However, according to the principle of

a Mingxia Zhao:879527755@qq.com 
relative motion, it was assumed that the epicyclical gear system was provided with a common angular velocity of "-WH" so that it revolved around the fixed axis of the planet carrier, at which the relative motion between the various components would remain unchanged, but the angular velocity became $\mathrm{WH}-\mathrm{WH}=0$, namely, the planet carrier could "stands still". Then the epicyclical gear train was converted into a fixed axis gear train, and the transmission ratio calculation formula of each pair of meshed gears in the gear train needed to be changed accordingly, which was as shown in equation 2[4-6].

$$
i_{j, j+1}=\frac{W_{j}-W_{H}}{W_{j+1}-W_{H}}= \pm \frac{z_{j+1}}{z_{j}}
$$

For the plane fixed gear train, the determination of transmission ratio "+" and "-" could be obtained by multiplying the positive and negative of each pair of meshing gear ratios. For space gear transmission, such as bevel gear, helical gear transmission, and so on; its total transmission ratio could be calculated using the above equation (2), but the plus-minus sign could only be used to draw the arrow to determine. According to the above equations (1) and (2), the transmission speed of the compound gear train in the paper was $\mathrm{W} 1=19 \mathrm{r} / \mathrm{min}$. The rotation speed in UG was ${ }^{\circ} / \mathrm{s}$ units, which was W1 $=19 * 360 / 60=114^{\circ} / \mathrm{s}$ after unit conversion.

\subsection{Transmission speed calculation of fixed axis gear train (a)}

The fixed shaft gear train (a) consisted of a bevel gear, which was a space gear transmission. The positive and negative of the total transmission ratio were marked by the arrow method. It could be seen from figure 1 that when the same direction was positive, and the calculation formula was as shown in equation (3).

$$
\left.\begin{array}{c}
\frac{W_{1}}{W_{2}}=-\frac{Z_{2}}{Z_{1}} \\
W_{2}=W_{2^{\prime}} \\
\frac{W_{2^{\prime}}}{W_{3}}=-\frac{Z_{3}}{Z_{2^{\prime}}}
\end{array}\right\} \Rightarrow \frac{W_{1}}{W_{2}}=\frac{Z_{2} \cdot Z_{3}}{Z_{1} \cdot Z_{2^{\prime}}} \Rightarrow W_{3}
$$

\subsection{Transmission speed epicyclical gear train (b)}

calculation

It was the plane fixed shaft gear train after the epicyclical gear train was converted into the fixed axis gear train. The transmission ratio of each pair of gears in the gear train was given by equation (4), which gave the formula for calculating the total transmission ratio. The absolute speed of some gears in the compound gear train was calculated by according to the characteristics of UG motion simulation and the principle of gear train transmission. The speed of meshing rotation of other gears could be achieved through the establishment of gear pairs to achieve speed transmission, so as to improve work efficiency.

$$
\begin{aligned}
& \frac{W_{3^{\prime}}-W_{H}}{W_{4}-W_{H}}=-\frac{Z_{4}}{Z_{3^{\prime}}}
\end{aligned}
$$



$$
\begin{aligned}
& \frac{W_{5^{\prime}}-W_{H}}{W_{6}-W_{H}}=-\frac{Z_{6}}{Z_{5^{\prime}}} \\
& W_{5}=W_{5}
\end{aligned}
$$

\section{Three dimensional modeling and assembly of compound gear train}

The gear tool box was used to carry out 3D modeling and assembly of the gear train by according to the gear mechanism sketch and gear parameters. Gear module took $\mathrm{m}=2.5$, gear modeling was completed by using modeling tools for spur gears and bevel gears, respectively; the tools included that the functions of creating gear, modifying the gear parameters, meshing gear, moving gears, deleting gears and other functions, it switched between different functions to achieve gear modeling and assembly, which could improve work efficiency.

\subsection{Modeling and assembly of cylindrical gear}

In UG, we should click the GC Toolbox $\rightarrow$ Gear Modeling $\rightarrow$ Spur Gears, select Create Gear Function, pop-up dialog box of involute cylindrical gear modeling, select parameter settings of the spur gear, external (internal) meshing gear and hobbing gear, and input the parameters such as modulo, number of gear, gear width and pressure angle after determining; we could also click the default value, the software would automatically add parameters, thus modify other parameters on the above basis; the vectors and points respectively refer to the direction of the axis of the spur gear and the center of the bottom surface; furthermore, we, respectively, needed to directly select the vector symbol and any point in the drawing area.

In the UG function area, we should switch to the meshing gear function, be set to engage the gear in all available list gears, click directly to set the driving and driven gear, where the driving, driven gear and mechanical principle refers to the driving, driven gear meaning, it was different. In UG, the driving gear referred to the gear that did not move the position during the modeling process; the driven gear referred to the gear whose position was moving, namely, when the gear was meshed, the driven gear should move position and close to the driving gear so as to mesh together. The center line vector was the vector that the center of the driving gear point to the center of the driven gear. According to the gear assembly position, we should select the corresponding vector to complete the assembly. According to the assembly position, we should also set the driving and driven gears as well as the center vector flexibly, so as to achieve the goal of successfully completing the gear assembly. 


\subsection{Modeling and assembly of bevel gear}

It was the same operation for bevel gear and spur gear, we should select the spur gear and equal head clearance shrinkage gear, also choose the default value for bevel gear parameters, just modify section cone angle; angle between the two axes was $\Sigma=90^{\circ}$ when the bevel gear were meshing, The sub-cone angle of gear 1 was calculated as equation $\delta_{1}=\arctan \left(Z_{1} / Z_{2}\right)$ and the subcone angle of gear 2 was equation $90-\delta_{1}$. In our case, the meshing of the bevel gear 1 and 2 could use the expression function to complete the sub-cone angle calculation. $\delta 1$ could be directly input when the parameter was modified; the other sub-cone angle of the meshing gear should be loaded $90-\delta 1$. We should switch to the gear meshing function, so that the driving and driven gear had the same meaning with cylindrical gear, driven gear axial vector was determined by according to the driven gear axis and assembly position, which was different from the cylindrical gear.

\section{Motion simulation of compound gear train}

\subsection{Creation of connecting rod and kinematic pair}

We accessed motion module in UG, where build a motion analysis program. The 22 connecting rods were created in the compound gear train, namely, a gear 1 , a double gears 2-2 ', a double gears 3-3', the 6 planetary gears 4 , the 6 planetary double gears 5-5 ', a planet carrier $\mathrm{H}$-gear 8 , a ring gear 6 , the 5 gears 7 .

\subsubsection{Rotation pair of fixed axis gear train}

The gear rotated about a fixed axis of rotation in the fixed axis gear train, it was relative to the ground movement, and that is, we needed establish a fixed rotating pair in UG, and did not need to select the relative bar. The fixed rotation pair was comprised of a gear 1, a double gears 2-2 ', a gear 7 and a planet carrier H-gear 8, which were denoted R1, R2, R7 and RH, respectively.

\subsubsection{Rotation pair of epicyclical gear train}

The epicyclical gear train included basic components such as a sun gear, a planet gear and a planet carrier. Both the sun gear and the planet carrier rotated about a fixed axis, and the planet gear and planet carrier were connected. On the one hand, they revolved around their own axis for rotation; on the other hand, they revolved around the axis of the planet carrier for revolution. Therefore, the planetary gear movement was the synthetic movement of the rotation and revolution, namely, we needed set in the UG relative rotation pair, select a planet carrier $\mathrm{H}$ which was relative rod, the rotation pair of the planet carrier $\mathrm{H}$ rotated to the ground; after planet with it as the relative rod, it was necessary to make a revolution together with the planet carrier, but also have a rotation of their selves. Relative to the planet carrier H-gear 8, the total of the rotation pairs was 14, which included a double gears 3-3', the 6 gears 4 , the 6 double gears 5-5' and a ring gear 6, it was denoted R3, R4, R5, R6, respectively; the relative rod of these 14 rotation pairs was a planet carrier $\mathrm{H}$-gear 8 , the second rod in the relative rod could be directly selected.

\subsubsection{Gear pair}

In the process of simulation of UG motion, the gear pair was used to establish the speed relationship between two rotation pairs, and the two rotation pairs must be relative to the same rod. When the gear pair was established, the first and second rotation pairs were sequentially selected, its ratio was the ratio of the number of the first rotation pair to the number of the second rotation pair, wherein the ratio of external gear was positive and the ration of internal gear was negative. we build the 19 gears ,that is, a gear 1 and a double gears 2-2 'were the ground rotation pair, the establishment of a gear pair, the ratio of $90 / 60$, which was denoted as G1; a dual gears 3-3' and a gear 4 were relative to the planet carrier $\mathrm{H}$, the establishment of 6 gears, the ratio of 24/18, which was denoted G2; Similarly, the 6 gears were established between a gear 4 and a double gears 5-5 ',the ratio of $18 / 15$, which was denoted by G3 ; a gear pair was established between a double gears 5-5 'and the ring gear 6 , a ratio of $-30 / 105$, which was denoted as G4, but it only needed to establish a gear pair between the two; the 6 double gears 5-5 ' were obtained the correct speed of engagement by G3 gear pair, which needed to selected a double gears 5-5' and a gear ring 6 to establish a gear pair, so that could enough a gear ring 6 to get the correct speed of engagement; therefore, we did not need to repeat the establishment, and repeating the establishment would cause the solver that could not solve and exercise simulation; the planet carrier H-gear 8 and the 5 gears 7 were the ground rotation pair, the establishment of gear 5 , the ratio of 35/32, which was denoted as G5. Rotation pair and gear pair of epicyclical gear train and fixed axis gear train in the compound gear train were shown in Figure 2.

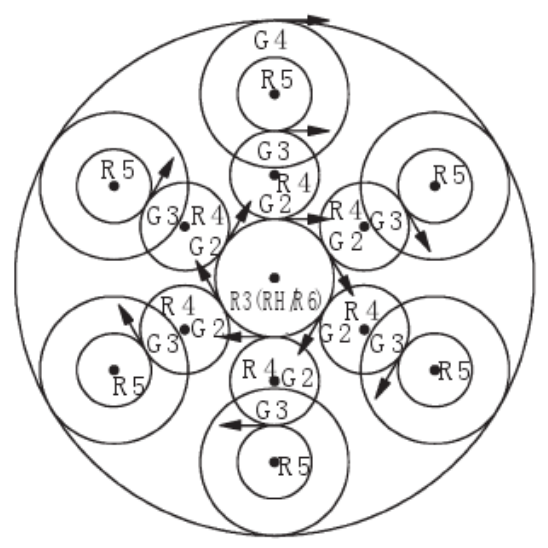




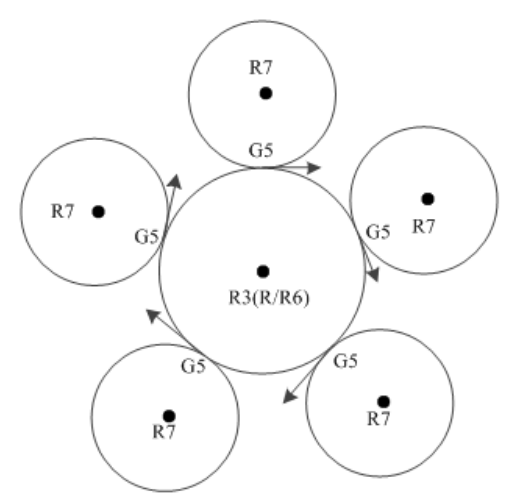

Figure 2. A schematic diagram of rotation pair and gear pair

\subsection{Definition of motion driven}

The gear pair could not define motion driven and limitation, which was just a speed transmission. The gear pair had been established by two gear pairs, one of them must have driven speed. The correct meshing rotation speed could be transmitted to the other rotation pair after establishing gear pair, so that we completed the simulation of gear movement. According to the design requirement, the constant driven speed of the rotation pair R1 of the gear 1 was $W_{l}=114 \%$, the speed of the rotation pair R3 of the double gear 3-3 'relative to the planet carrier $\mathrm{H}$ was $W_{3}-W_{H}=171+144=315 \%$, the speed of rotation pair of planet carrier $\mathrm{H}$-gear 8 was $R_{H}$ $=-144^{\circ} / \mathrm{s}$.

\subsection{Motion Simulation}

A motion simulation solution was set up in the analysis process of tool bar, in which the solution type was conventional driven and the analysis type was kinematics or dynamics, the time was set as $2 s$, the number of steps was 300; ADAMS solver process would been activated after you clicked OK button. Animation tool would been started to see the movement process of entire compound gear train, you could also make the motion simulation process into MPEG movie files, or the various components of the gear train movement was directly expressed to the electronic chart.

The relationship between the absolute speed and the time of each rotation pair in the compound gear train was shown as figure 3 .

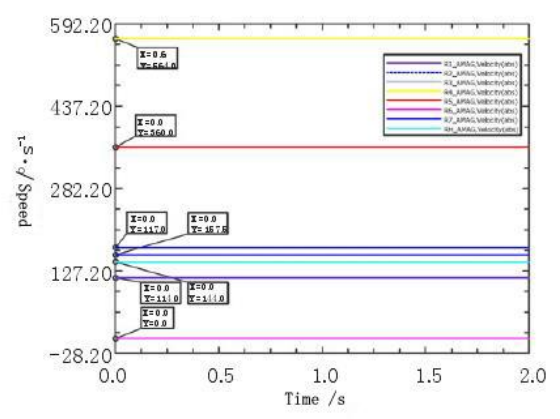

Figure3. The relationship between the absolute speed and the time of gear train
It could be seen from FIG. 3 that all the gears in the compound gear train were driven by the gear 1 of input, and all the components rotated at a constant angular velocity. There was no mutual collision and mutual stagnation among the various mechanisms and could also be observed in the animated process of motion simulation. the double gears 2-2 'and the 6 double gears $3-3$ ' had the same angular velocity of $171 \%$ rotation; the 6 intermediate gears 4 and the 6 intermediate double gears 5-5 ' had $564 \%$ and $360 \%$ angular velocity, respectively; the sun gear ring 6 speed was $0^{\circ} / \mathrm{s}$, which was fixed to the bracket; the planet carrier H-gear 8 had $144^{\circ} / \mathrm{s}$ angular velocity rotation; the gear 7 had $157.5 \%$ angular velocity rotation. The transmission ratio of compound gear train $i_{17}=W_{1} / W_{7}=114 / 157.5=0.72$, which was in line with the design requirements of transmission ratio; the design of compound gear train gear was correct, and each component was relatively stable during movement, which met the assembly requirements.

\section{Conclusion}

The movement mechanism of the gear train was analyzed by applying the UG motion analysis module, which was possible to get an intuitive expression of the movement status of the gear train mechanism, and to clearly observe whether there was interference between the various components or not. The solution had the characteristics of being clear, effective, economical and quick. Depending on the scope of use and the purpose of analysis, we could set the vector torque and other driven, which might analyze the gear load and acceleration, and also connect with the finite element analysis module, which might analyze the finite element of gear; all the design improves work efficiency and reduced costs.

\section{References}

1. Broomhead D S, Lowe D. Multivariable functional interpolation and adaptive networks [J]. Complex System, 1988, 2(1): 321-355.

2. HL K, Neural P S. Network ensembles [J]. IEEE Transactions on Pattern Analysis and Machine Intelligence, 1990, 12(10): 993-1001.

3. Kennedy J, Eberhart R. Particle swarm optimization $[\mathrm{C}] / /$ Proceedings of IEEE International Conference on Neural Networks, Piscataway, 1995: 1942-1948.

4. Qin Zheng, Chen Junying, Liu Yu, et al. Evolving RBF neural networks for pattern classification $[\mathrm{C}] / /$ International Conference on Computational Intelligence and Security, China, 2005: 957-964.

5. Freund Y, Robert E S. A decision-theoretic generalization of on-line learning and an application to boosting [J]. Journal of Computer and System Sciences, 1997, 55(1): 119-139. 
6. Osada R, Funkhouser $\mathrm{T}$, Chazelle $\mathrm{B}$, et al. Matching 3D models with shape distributions $[\mathrm{C}] / /$ Proceedings of the International Conference on Shape Modeling and Applications, Genoa, 2001: 154-159 